\title{
The CUORICINO and CUORE experiments
}

\section{R. Ardito and G. Maier}

Dipartimento di Ingegneria Strutturale del Politecnico di Milano, Milano I-20133, Italy

C. Arnaboldi, C. Brofferio, S. Capelli, F. Capozzi, L. Carbone, O. Cremonesi,

E. Fiorini, A. Nucciotti, M. Pavan, G. Pessina, S. Pirro, E. Previtali, M. Sisti,

L. Torres, and L. Zanotti

Dipartimento di Fisica dell'Università di Milano-Bicocca e Sezione di Milano dell'INFN, Milano I-20126, Italy

D. R. Artusa, F. T. Avignone, I. Bandac, R. J. Creswick, H. A. Farach, and

\section{Rosenfeld}

Dept.of Physics and Astronomy, University of South Carolina, Columbia, South Carolina, USA 29208

M. Balata, C. Bucci, and M. Pyle Laboratori Nazionali del Gran Sasso, I-67010, Assergi (L'Aquila), Italy

M. Barucci, E. Olivieri, E. Pasca, L. Risegari, and G. Ventura Dipartimento di Fisica dell'Università di Firenze e Sezione di Firenze dell'INFN, Firenze I-50125, Italy

J. Beeman, R. J. McDonald, E. B. Norman, and A. R. Smith Lawrence Berkeley National Laboratory, Berkeley, California, 94720, USA

S. Cebrián*, P. Gorla, I. G. Irastorza† A. Morales, and C. Pobes Laboratorio de Física Nuclear y Altas Energías, Universidad de Zaragoza, E-50009 Zaragoza, Spain

A. de Waard and G. Frossati

Kamerling Onnes Laboratory, Leiden University, 2300 RAQ, Leiden, The Netherlands

A. Fascilla, A. Giuliani, and M. Pedretti

Dipartimento di Scienze Chimiche, Fisiche e Matematiche dell'Università dell'Insubria e Sezione di Milano dell'INFN, Como I-22100, Italy

E. E. Haller

Department of Materials Science and Engineering, University of California, Berkeley, California 94720, USA

\section{Palmieri}


Laboratori Nazionali di Legnaro, Via Romea 4, I-35020 Legnaro ( Padova ), Italy

\section{E. Guardincerri, P. Ottonello, and M. Pallavicini}

Dipartimento di Fisica dell'Università di Genova e Sezione di Genova dell'INFN, Genova I-16146, Italy

ABstract: CUORE is an experiment intended to investigate rare events like the double beta decay and the interaction of cold dark matter and solar axions. It consists of a tightly packed array of $1000 \mathrm{TeO}_{2}$ bolometers, having a mass of $750 \mathrm{~g}$ each, operating in the Laboratori Nazionali del Gran Sasso (LNGS) in Italy. A first step towards CUORE is CUORICINO, an experiment with $40.7 \mathrm{~kg}$ of $\mathrm{TeO}_{2}$ running since the beginning of 2003 in Gran Sasso. In this paper, the CUORE project is described, the first results of CUORICINO for the neutrinoless double beta decay of ${ }^{130} \mathrm{Te}$ are given, and the physics potential and prospects of both CUORICINO and CUORE are presented.

Dedicated to Angel Morales

\section{Introduction}

Neutrino oscillation experiments have unequivocally demonstrated that neutrinos have mass and that the neutrino mass eigenstates mix [i] valuable information on the mixing angles and on the mass differences of the three eigenstates. They cannot, however, determine the scale of the neutrino mass, which is fixed by the lightest neutrino mass eigenvalue. This can only be directly determined by beta decay end point spectral shape measurements, or in the case of Majorana neutrinos by the observation and measurement of the neutrinoless double beta decay half-life.

CUORE (Cryogenic Underground Observatory for Rare Events) is intended to investigate low probability events like double beta decay (DBD), operating a large array of bolometers of $\mathrm{TeO}_{2}$ underground in the Gran Sasso Laboratory (Italy). The first step of this project, called CUORICINO, is presently running in Gran Sasso. The main features

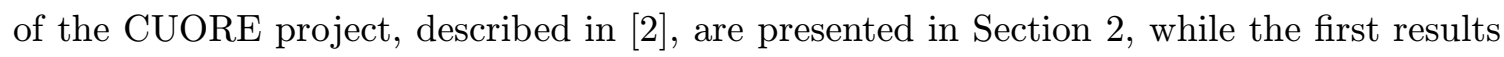

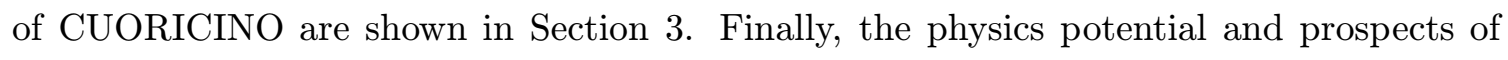
both experiments are discussed in Section 崔.

\section{The CUORE project}

\subsection{Why use bolometers of $\mathrm{TeO}_{2}$ ?}

A thermal detector measures the energy deposited by an interacting particle through the corresponding temperature rise, recorded by means of a proper thermal sensor. These

\footnotetext{
${ }^{*}$ Speaker.

${ }^{\dagger}$ Present address: CERN, Experimental Physics Division, CH-1211 Geneva 23, Geneva, Switzerland.
} 
detectors must have a very small heat capacity, which is accomplished by using suitable materials (dielectrics, superconductors below the phase transition, etc.) and by running the detector at very low temperatures (usually below $100 \mathrm{mK}$ ).

The use of cryogenic detectors to search for rare events was firstly proposed in 1984 [3]- This kind of detector has very good energy resolution and the advantage of very loose requirements on the crystal absorber choice, which favours their use in the source=detector approach, where the bolometer must be also the source of DBD. In addition, ${ }^{130}$ Te itself has other advantages when looking for the neutrinoless DBD: a high natural isotopic abundance (33.87\%), high transition energy $\mathrm{Q}=(2528.8 \pm 1.3) \mathrm{keV}$ (which means large phase space and low background since the neutrinoless DBD signal, a sharp peak at Q, is expected to appear in a clean window between full energy and Compton edge of ${ }^{208} \mathrm{Tl}$ photons), encouraging theoretical calculations for the neutrinoless channel lifetime and the fact that DBD has been already observed with geochemical techniques $\left(T_{1 / 2}^{i n c l}=(0.7-2.7) \times 10^{21}\right.$ y $)$.

\subsection{The MiDBD experiment}

The idea of CUORE proceeds from the successful results of MiDBD (see Ref. [4i and references therein), which can therefore be considered as a first step towards CUORE. This experiment, carried out in Gran Sasso by the Milano group, was only the last of a series of experiments on neutrinoless DBD of ${ }^{130} \mathrm{Te}$, based on the bolometric technique. The possibility to use the available MiDBD cryogenic system to test one of the 25 constitutive crystal towers of CUORE led to CUORICINO, presently running. The CUORE detector will therefore be the final version of a three step development comprising MiDBD, CUORICINO, and finally CUORE.

MiDBD consisted of an array of 20 crystals of $\mathrm{TeO}_{2}$ of $3 \times 3 \times 6 \mathrm{~cm}^{3}$ each $(340 \mathrm{~g})$, for a total mass of $6.8 \mathrm{~kg}$, operated until December 2001. Sixteen MiDBD crystals were made of natural $\mathrm{TeO}_{2}$. Of the remaining four, two were isotopically enriched at $82.3 \%$ in ${ }^{128} \mathrm{Te}$ and other two at $75.0 \%$ in ${ }^{130} \mathrm{Te}$. The second phase of the experiment (MiDBD-II) was devoted to check both the knowledge of the background sources and the performance of the new detector mounting system proposed for CUORICINO and CUORE. For this run the tower was completely rebuilt with a structure very similar to the one of CUORICINO (i.e. of the proposed CUORE towers).

The final results of the MiDBD experiment for different channels of DBD in ${ }^{130} \mathrm{Te}$ and ${ }^{128} \mathrm{Te}$ can be found in ${ }_{\mathrm{A}}$. No peak appeared in the region of $\beta \beta(0 \nu)$ of ${ }^{130} \mathrm{Te}$, where the background rate was of $0.33 \pm 0.11$ counts $\mathrm{keV}^{-1} \mathrm{~kg}^{-1}$ year ${ }^{-1}$; the corresponding limit for the lifetime was $\mathrm{T}_{1 / 2}>2.1 \times 10^{23}$ y $(90 \%$ C.L. $)$.

\subsection{CUORE detector}

The CUORE detector will consist of an array of $1000 \mathrm{TeO}_{2}$ bolometers arranged in a cubic configuration of 25 towers, in a 5 by 5 matrix. Each tower contains 10 four-crystal modules.

A single CUORE bolometer consists of a $5 \times 5 \times 5 \mathrm{~cm}^{3}$ single crystal of $\mathrm{TeO}_{2}$ having a mass of $750 \mathrm{~g}$, that acts both as detector and source; each single crystal is grown with ultrapure $\mathrm{TeO}_{2}$ powders and is optically polished on the surfaces. Crystals of $\mathrm{TeO}_{2}$ have a tetragonal structure, and are grown along the (001) axis. The temperature changes will 


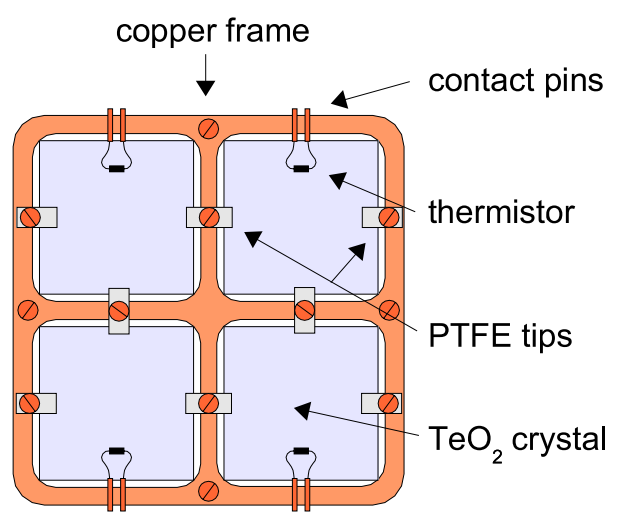

Figure 1: A four detector module.

be recorded using Neutron Transmutation Doped (NTD) germanium thermistors attached by epoxy to the crystal. These devices have been made uniquely uniform in their response and sensitivity. A Si doped resistor is also glued on the surface of each crystal, acting as a heater to generate a reference heat pulse in the crystal in order to monitor continuously

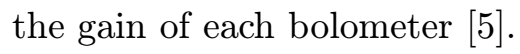

The structure of each four-detector module is shown in Fig. held between two copper frames joined by copper columns. There is a $6 \mathrm{~mm}$ gap between crystals with no material between them. PTFE (Polytetrafluoroethylene or TEFLON) pieces are inserted between the copper and $\mathrm{TeO}_{2}$, as a heat impedance to delay the recooling of the bolometers, and to clamp the crystals.

\subsection{CUORE set-up}

The array of CUORE towers will be suspended from a large square copper plate thermally connected to the mixing chamber of the dilution refrigerator. The suspension will be made by a vertical spring to decouple the detector from the vibrations of the dilution refrigerator [6]. CUORE will operate at temperatures between 7 and $10 \mathrm{mK}$. This will require an extremely powerful dilution refrigerator. $\mathrm{A} \mathrm{He}^{3} / \mathrm{He}^{4}$ refrigerator with a cooling power of 3 $\mathrm{mW}$ at $120 \mathrm{mK}$ will be used.

The problem of shielding and of using only low radioactive contamination materials is particularly complex when using complicated structures like dilution refrigerators. In CUORE, part of the bulk lead shielding will be placed inside the cryostat, and part outside (see Fig. (2) $4 \mathrm{mBq} / \mathrm{kg}$ ) will constitute $3 \mathrm{~cm}$-thick walls of the cubic structure of the array. The top of the detector array will be protected by two $10 \mathrm{~cm}$-thick lead layers $1 \mathrm{~m}$ by $1 \mathrm{~m}$, with a ${ }^{210} \mathrm{~Pb}$ activity of $16 \mathrm{~Bq} / \mathrm{kg}$ (the inner layer) and $150 \mathrm{~Bq} / \mathrm{kg}$ (the outter one). Another layer of low-activity lead, $17 \mathrm{~cm}$ thick, and $40 \mathrm{~cm}$ by $40 \mathrm{~cm}$ will be placed directly on the top face of the detector. Finally, outside the dewar, there will be two $10 \mathrm{~cm}$ thicknesses of lead, $16 \mathrm{~Bq} / \mathrm{kg}$ of ${ }^{210} \mathrm{~Pb}$ for the inner layer, and $150 \mathrm{~Bq} / \mathrm{kg}$ for the outer layer. The lead shield will be surrounded with a $10 \mathrm{~cm}$ thick box of borated polyethylene that will also function as an hermetically sealed enclosure to exclude radon. It will be flushed constantly with dry nitrogen. 


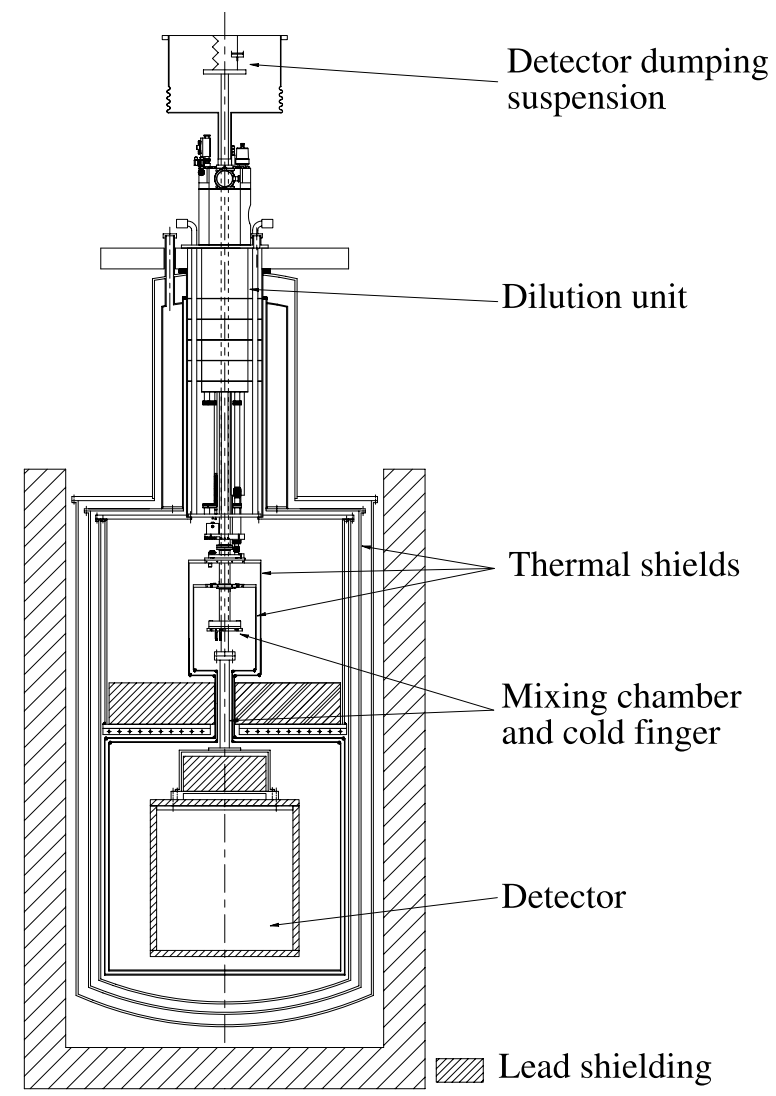

Figure 2: CUORE cryostat and shielding.

The entire dewar, detector, and shield will be enclosed in a Faraday cage to exclude electromagnetic disturbances that also constitute a source of background, albeit at low energies. The addition of a muon veto surrounding the entire structure is also being considered. CUORE will be located in the underground halls of LNGS at a depth of 3400 mwe.

\section{First results of CUORICINO}

The CUORICINO detector is a CUORE-like tower with 13 planes. Eleven of these planes are 4-crystal modules identical to those foreseen for CUORE. Two additional planes are made of 9-crystal modules. In these two planes 18 of the 20 crystals used for the MiDBD experiment have been housed. CUORICINO contains therefore $44 \mathrm{TeO}_{2}$ crystals of about $5 \times 5 \times 5 \mathrm{~cm}^{3}$ and average mass $790 \mathrm{~g}$ and $18 \mathrm{TeO}_{2}$ crystals of about $3 \times 3 \times 6 \mathrm{~cm}^{3}$ and average mass $330 \mathrm{~g}$. The total mass of $\mathrm{TeO}_{2}$ in CUORICINO is $40.7 \mathrm{~kg}$. All the crystals are made of natural tellurium but the 4 isotopically enriched crystals of MiDBD.

CUORICINO was cooled at the beginning of 2003. Unfortunately during the cooling procedure some of the signal wires disconnected so that only 32 of the large size crystals and 16 of the small ones could be read. The technical problem responsible for the disconnection 


\begin{tabular}{ccccc}
\hline \hline counts $/ \mathrm{keV} / \mathrm{kg} /$ year & $1-2 \mathrm{MeV}$ & $2-3 \mathrm{MeV}$ & $3-4 \mathrm{MeV}$ & $4-5 \mathrm{MeV}$ \\
\hline MiDBD-II & $3.21 \pm 0.08$ & $0.61 \pm 0.04$ & $0.29 \pm 0.02$ & $1.88 \pm 0.06$ \\
$3 \times 3 \times 6$ natural & $3.29 \pm 0.11$ & $0.38 \pm 0.04$ & $0.24 \pm 0.03$ & $0.78 \pm 0.05$ \\
$5 \times 5 \times 5$ & $3.25 \pm 0.09$ & $0.41 \pm 0.03$ & $0.17 \pm 0.02$ & $0.55 \pm 0.03$ \\
\hline \hline
\end{tabular}

Table 1: Counting rates per unit mass in MiDBD-II and in CUORICINO.

of the wires is now identified and will be repaired. The performance of the electrically connected detectors is quite good: the average FWHM resolution at $2615 \mathrm{keV}$ measured during the calibration of the detectors with a ${ }^{232} \mathrm{Th}$ source is of $\sim 7 \mathrm{keV}$ for the $5 \times 5 \times 5$ $\mathrm{cm}^{3}$ crystals and of $\sim 9 \mathrm{keV}$ for the $3 \times 3 \times 6 \mathrm{~cm}^{3}$ crystals.

On April 19th the first background measurement started and was over on July 21th due to the interruption of the water supply by the LNGS. During this run the live time was $\sim 72 \%$.

\subsection{CUORICINO background}

The total collected statistics are therefore of $\sim 25000$ hours $\times \mathrm{kg}$ for $5 \times 5 \times 5 \mathrm{~cm}^{3}$ crystals and $\sim 2300$ hours $\times \mathrm{kg}$ for $3 \times 3 \times 6 \mathrm{~cm}^{3}$ ones. The gamma lines due to ${ }^{60} \mathrm{Co},{ }^{40} \mathrm{~K}$ and of the ${ }^{238} \mathrm{U}$ and ${ }^{232} \mathrm{Th}$ chains are clearly visible. Also visible are the gamma lines due to Te activation $\left({ }^{121} \mathrm{Te},{ }^{121 m} \mathrm{Te},{ }^{123 m} \mathrm{Te},{ }^{125 m} \mathrm{Te}\right.$ and $\left.{ }^{127 m} \mathrm{Te}\right)$ and those due to $\mathrm{Cu}$ activation $\left({ }^{57} \mathrm{Co},{ }^{58} \mathrm{Co}\right.$, ${ }^{60} \mathrm{Co}$ and ${ }^{54} \mathrm{Mn}$ ). The background in the neutrinoless DBD region (i.e. $2510-2580 \mathrm{keV}$ ) is $0.20 \pm 0.03$ counts $/ \mathrm{keV} / \mathrm{kg} / \mathrm{y}$ for the $5 \times 5 \times 5 \mathrm{~cm}^{3}$ crystals and $0.2 \pm 0.1$ counts $/ \mathrm{keV} / \mathrm{kg} / \mathrm{y}$ for the $3 \times 3 \times 6 \mathrm{~cm}^{3}$ ones.

Because of the low collected statistics, any evaluation of the possible background sources is still preliminary. General shapes and counting rates (when normalized to the mass of the crystals) are quite similar in large and small size crystals but the intensities of the gamma lines do not show a clear behaviour. In Table $\underline{1}_{1}^{1}$ the counting rates in different energy regions are reported. It is clearly evident that an improvement was obtained in the 2-3 MeV region (a reduction of $\sim 30 \%$ in the counting rates per unit mass either of the small and of the big crystals) despite the reduced internal lead shield used in CUORICINO ( $\sim 1.2 \mathrm{~cm}$ thickness of roman lead) with respect to MiDBD-II $(\sim 3 \mathrm{~cm}$ thickness of roman lead). An even better reduction was obtained in the $3-4 \mathrm{MeV}$ region where background is mainly dominated by surface contamination contributions.

\subsection{Neutrinoless double beta decay results}

No peak appears in the anticoincidence background spectrum at the ${ }^{130} \mathrm{Te}$ DBD transition energy (see Fig. $\left.\underline{3}_{i}\right)$. A Maximum Likelihood procedure used to establish the maximum number of $\beta \beta(0 \nu)$ events compatible with the measured background allows to set a lower limit on the ${ }^{130} \mathrm{Te} \beta \beta(0 \nu)$ half-life of $5.5 \times 10^{23}$ years at $90 \%$ C.L. The corresponding upper bound on the Majorana effective mass ranges from 0.37 and $1.9 \mathrm{eV}$ depending on the nuclear model used to interpret the data (see Table $\left.\overline{2}_{1}^{1}\right)$. QRPA (Quasiparticle Random Phase Approximation) models seem the most/only suitable for heavy nuclei. 
Table 2: Upper limits (90\% C.L.) on $\left\langle m_{\nu}\right\rangle$ from CUORICINO result $\left(\mathrm{T}_{1 / 2}>5.5 \times 10^{23} \mathrm{y}\right)$ for different QRPA evaluations.

\begin{tabular}{|c|c|c|c|}
\hline Authors/Ref. & $\left\langle m_{\nu}\right\rangle(\mathrm{eV})$ & Authors/Ref. & $\left\langle m_{\nu}\right\rangle(\mathrm{eV})$ \\
\hline Staudt et al. $\left[\overline{7_{1}}\right]$ & $0.37-0.4$ (pairing, Paris) & Suhonen et al. [1] $\left[\begin{array}{l}1 \\
1\end{array}\right]$ & 1.5 \\
\hline & 0.4-0.44 (pairing, Bonn) & Muto et al. [1] $\left[\begin{array}{l}1 \\
1\end{array}\right]$ & 0.93 \\
\hline Pantis et al. [isin] & $\begin{array}{c}1.2 \text { (no p-n pairing) } \\
1.9 \text { (p-n pairing })\end{array}$ & Stoica et al. $[1]$ & $\begin{array}{l}1.4 \text { (large basis) } \\
1.3 \text { (short basis) }\end{array}$ \\
\hline Vogel $[\overline{\bar{p}}]$ & 1.1 & Faessler et al. [1] & 1.3 \\
\hline Citivarese [is $\left[\begin{array}{l}1 \\
2\end{array}\right]$ & 0.97 & Engel et al. [i] $[\overline{1} \overline{1}]$ & 0.66 (seniority) \\
\hline Tomoda [1] & 0.97 & Aunola et al. $[1 \overline{1} \overline{9}]$ & $0.91(\mathrm{WS})$ \\
\hline Barbero et al. [1] & 0.78 & & 0.97 (AWS) \\
\hline Simkovich [1] $\overline{3} \overline{3}]$ & $1.6(\mathrm{pn}-\mathrm{RQRPA})$ & & \\
\hline
\end{tabular}

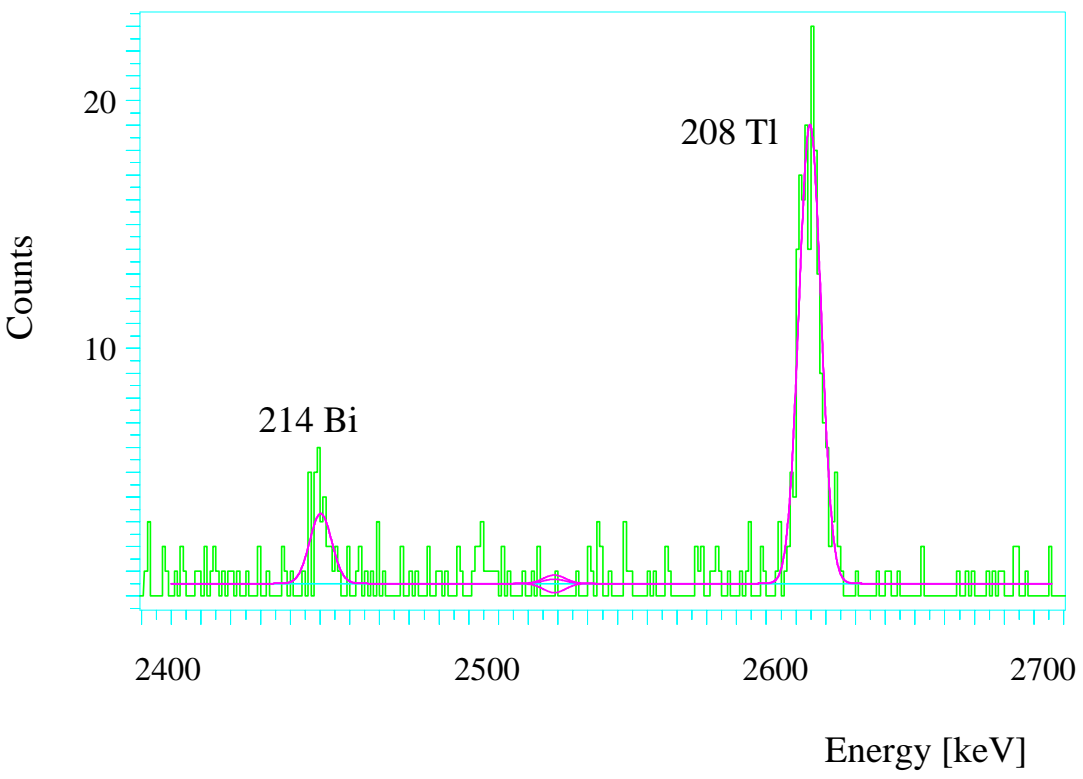

Figure 3: Summed background spectrum from all the operating crystals in the region of neutrinoless DBD of ${ }^{130} \mathrm{Te}(\mathrm{Q}$-value $=2528.8 \mathrm{keV})$.

The early CUORICINO data already show that CUORICINO is a competitive experiment in the field of neutrinoless DBD. Once completely working CUORICINO will have a 5 year sensitivity (at $68 \%$ C.L.) of about $10^{25}$ year for the $\beta \beta(0 \nu)$ of ${ }^{130} \mathrm{Te}$. This means that CUORICINO will be able to test the Majorana mass in the 100-500 meV range, improving even the upper bounds set by the ${ }^{76} \mathrm{Ge}$ experiments. 


\section{Physics Potential and Prospects}

A Monte Carlo study has been carried out to estimate the achievable background levels in CUORE. Assuming the $90 \%$ upper limits on bulk contaminations measured in the MiDBD experiment, and a reduction by a factor 20 of the crystal and copper surface contamination, it has been estimated that the CUORE background could be $\sim 0.007$ counts/(keV kg y) at the $\beta \beta(0 \nu)$ transition energy and $\sim 0.05$ counts $/(\mathrm{keV} \mathrm{kg} \mathrm{d})$ near threshold. With these hypotheses CUORE is a powerful instrument to search for DBD and dark matter. However, the goal of the CUORE technical $R \& D$ of the next few years will be the reduction of background to the level of 0.001 counts $/(\mathrm{keV} \mathrm{kg} \mathrm{y)}$ at the $\beta \beta(0 \nu)$ transition energy and to 0.01 counts $/(\mathrm{keV} \mathrm{kg} \mathrm{d})$ at threshold. A complete discussion of the physics potential and prospects of CUORICINO and CUORE can be found in [2] $\overline{2} \overline{1}]$.

\subsection{Double Beta Decay}

As pointed out before, ${ }^{130} \mathrm{Te}$ is a good candidate for double beta decay searches. The nuclear factor-of-merit $F_{N}$ (depending on a kinematical phase space factor and on nuclear matrix elements) is a factor $5-10$ more favorable for ${ }^{130} \mathrm{Te}$ than for ${ }^{76} \mathrm{Ge}$, atom for atom, which translates into a factor 2 to 3 better as far as the $\left\langle m_{\nu}\right\rangle$ bounds are concerned.

The detector factor-of-merit $F_{D}^{0 \nu}$, or detection sensitivity, provides an approximate estimate of the neutrinoless half-life limit achievable with a given detector. For source=detector devices, it reads:

$$
F_{D}^{0 \nu}=4.17 \times 10^{26} \times \frac{a}{A} \sqrt{\frac{M t}{b \Gamma}} \times \epsilon \quad \text { years }
$$

where $A$ is the atomic mass, $a$ is the isotopic abundance, $M$ the detector mass in $\mathrm{kg}$, $b$ the background in $\mathrm{c} / \mathrm{keV} / \mathrm{kg} / \mathrm{y}$ in the neutrinoless DBD region, $t$ the running time in years, $\Gamma$ the FWHM energy resolution in $\mathrm{keV}$ and $\epsilon$ the detector efficiency (which is 0.86 in the case of the cubic $5 \times 5 \times 5 \mathrm{~cm}^{3}$ crystals). In the case of a $\mathrm{TeO}_{2}$ crystal detector, $F_{D}^{0 \nu} \sim 7.59 \times 10^{23} \sqrt{\frac{M t}{b \Gamma}}$, with $M$ the crystal mass in $\mathrm{kg}$ and $b$ the background in counts per $\mathrm{keV}$ per year and per $\mathrm{kg}$ of detector mass.

CUORICINO sensitivity has been already presented in Section 3 full CUORE physics potential, $\mathrm{F}_{D}$ can be evaluated according to our projection. Assuming a background of $\mathrm{b}=0.01 \mathrm{c} / \mathrm{keV} / \mathrm{kg} / \mathrm{y}$ and an energy resolution $\Gamma(2.5 \mathrm{MeV})=5 \mathrm{keV}$, we would get $\mathrm{F}_{D}^{0 \nu}$ of $9.4 \times 10^{25} \sqrt{t}$ years $\left(6.5 \times 10^{25} \sqrt{t}\right.$ years for $\left.\Gamma=10 \mathrm{keV}\right)$, which in t years of statistics would provide $\left|\left\langle m_{\nu}\right\rangle\right|$ bounds in the range 0.036-0.2 $\mathrm{t}^{-1 / 4} \mathrm{eV}$ (according to the predictions of $\mathrm{F}_{N}$ of QRPA models). However, the R\&D to be carried out in CUORE, if successful, would provide a value of $\mathrm{b} \sim 0.001 \mathrm{c} / \mathrm{keV} / \mathrm{kg} / \mathrm{y}$, i.e. a detection sensitivity of $\mathrm{F}_{D} \sim 2.96 \times 10^{26} \sqrt{t}$ years $\left(2.1 \times 10^{26} \sqrt{t}\right.$ years for $\left.\Gamma=10 \mathrm{keV}\right)$, or $\left|\left\langle m_{\nu}\right\rangle\right|$ bounds in the range $\sim 0.02-0.11 \mathrm{t}^{-1 / 4} \mathrm{eV} . \mathrm{TeO}_{2}$ crystals made with ${ }^{130} \mathrm{Te}$ enriched material have been already operated in MiDBD and CUORICINO, making an enriched CUORE a feasible option. Assuming a $95 \%$ enrichment in ${ }^{130} \mathrm{Te}$ and a background level of $\mathrm{b}=0.001 \mathrm{c} / \mathrm{keV} / \mathrm{kg} / \mathrm{y}$, the sensitivity would become $\mathrm{F}_{D} \sim 8.32 \times 10^{26} \sqrt{t}$ years. For an exposure of 5 years, the 
corresponding $\left|\left\langle m_{\nu}\right\rangle\right|$ bounds would range from $8 \mathrm{meV}$ to $45 \mathrm{meV}$ depending on the nuclear matrix element calculations.

\subsection{WIMP detection}

The direct detection of dark matter particles like WIMPs (Weakly Interacting Massive Particles) relies on the measurement of their elastic scattering off target nuclei in a suitable detector. To claim a positive identification a distinctive signature like the annual modulation effect [2]2̄i] is needed. The DAMA Collaboration has reported a seasonal effect

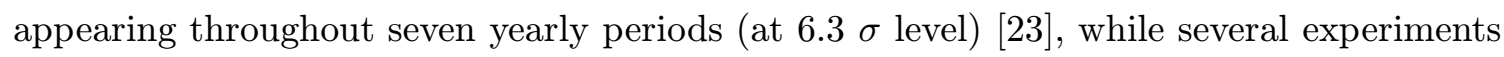
[2] framework of standard models.

The capabilities of CUORICINO and CUORE to exclude or detect WIMPs have been evaluated. In Fig. 'i-i (left), the expected exclusion plots for coherent spin-independent WIMP-matter interaction are shown for two possible values of the background of CUORICINO, 1 and $0.1 \mathrm{c} / \mathrm{keV} / \mathrm{kg} /$ day. The first value is of the order of the background already achieved from threshold onwards $(10-50 \mathrm{keV})$ in the MiDBD latest results (see [205i]). A low energy resolution of $1 \mathrm{keV}$ and an energy threshold of $10 \mathrm{keV}$ have been assumed as well as an exposure of 2 years of CUORICINO ( $81 \mathrm{~kg} \cdot$ year). The projected exclusion contours are compared with the one currently obtained from MiDBD (dashed line) [2.25] and with the $3 \sigma$ CL region of WIMPs singled out by the modulation analysis performed by the

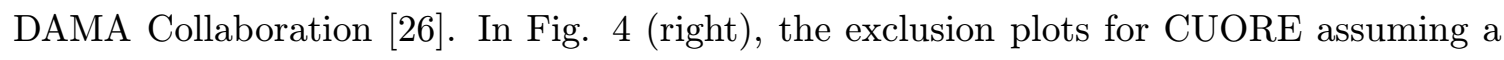
background of 0.05 and $0.01 \mathrm{c} / \mathrm{keV} / \mathrm{kg} /$ day, are similarly presented for an exposure of 1 year (750 kg.year).
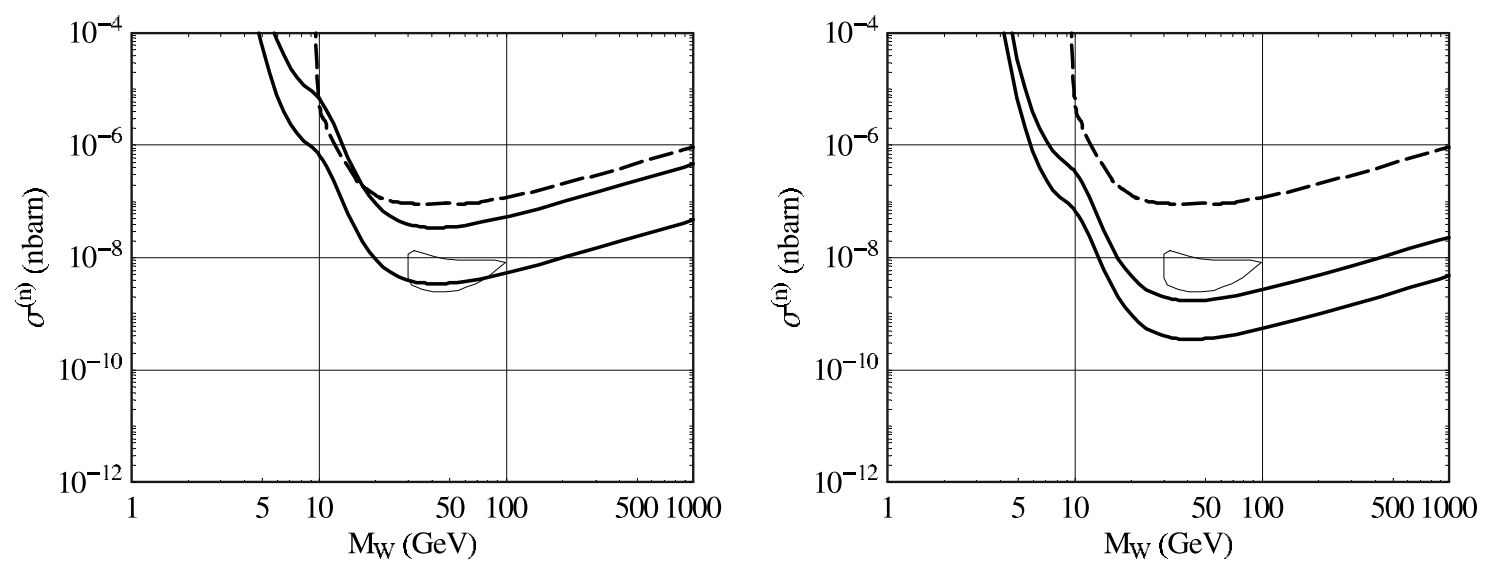

Figure 4: Exclusion plots projected for CUORICINO (left) and CUORE (right) (see text). The closed curve represents the DAMA region. The dashed line corresponds to the current MiDBD result.

CUORE and to some extent CUORICINO have detector masses large enough to search for the annual modulation signal. An essential requirement to search for seasonal effects is to have a superb control of systematic errors and to assure that the stability of the various experimental parameters, which might mimic periodic variations of the signals, are kept within a small fraction of the (already tiny) expected signal. Assuming that all these 
fluctuations are controlled well below the levels needed $(<1 \%)$, one can proceed to analyze the sensitivity of CUORICINO/CUORE to the annual modulation signal.

The sensitivity of a given experimental device to this annual modulation signal depends on the detector material employed and the experimental parameters of the detectors; it has

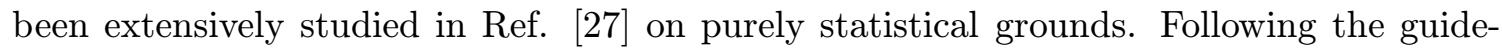
lines of that reference, we have estimated the region of WIMPs that could be within reach for CUORE and CUORICINO with the above mentioned assumptions on the background levels. We have fixed a sensitivity condition that corresponds to $50 \%$ probability of obtaining a positive modulation signal at $90 \%$ C.L.. In Fig. $\underline{\underline{s}}_{1}$ (left) curves are shown obtained for a threshold of $10 \mathrm{keV}$, two years of exposure with CUORICINO (81 kg year) and two assumed flat backgrounds of 1 and $0.1 \mathrm{c} / \mathrm{keV} / \mathrm{kg} /$ day. One can see that CUORICINO could already explore most of the DAMA region looking for a positive annual modulation signal. In Fig. and $0.01 \mathrm{c} / \mathrm{keV} / \mathrm{kg} /$ day, two years of exposure of CUORE (1500 kg year) and a threshold of $10 \mathrm{keV}$ (thick solid lines); in this case, the possibility of a lower thresholds of $5 \mathrm{keV}$ with a background of $0.01 \mathrm{c} / \mathrm{keV} / \mathrm{kg} /$ day is also shown (dashed line).
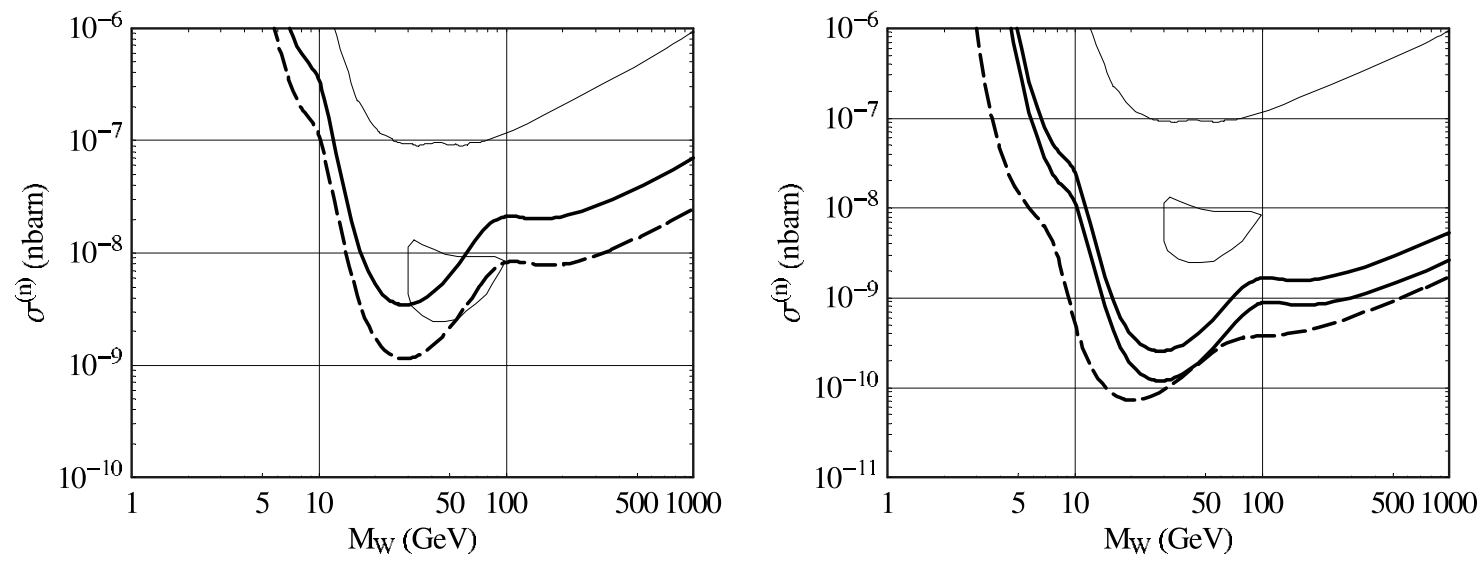

Figure 5: Sensitivity plots in the $(m, \sigma)$ plane for CUORICINO (left) and CUORE (right) (see text). The closed contour represents the DAMA region and the thin solid line corresponds to the current MiDBD exclusion.

\subsection{Solar axion detection}

Detectors of single crystals provide a simple mechanism for solar axion detection based on the Bragg coherent Primakoff conversion [i2 $\overline{2} \overline{1}]$. This method has already been employed

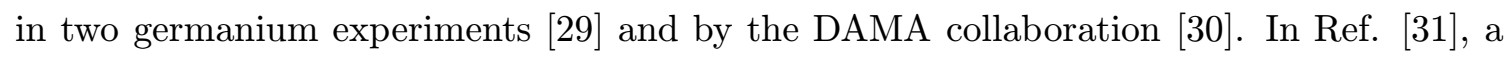
calculation of the perspectives of various crystal detectors for solar axion searches has been made.

The use of CUORE to search for solar axions should have a priori some advantages with respect to germanium detectors, because of the higher $\mathrm{Z}$, larger mass and known orientation of the crystals. Accordingly, as the cross-section for Primakoff conversion depends on the square of the atomic number, $\mathrm{TeO}_{2}$ will be a priori a better candidate than Germanium. A low energy threshold is mandatory because the expected signal lies in the energy region 
$2 \mathrm{keV} \lesssim E \lesssim 10 \mathrm{keV}$ and peaks at $E \simeq 4 \mathrm{keV}$. Following Ref. [3ì] photon coupling which can be achieved in CUORICINO and CUORE has been estimated for some assumed values of the experimental parameters. The results are shown in Table i3i. In all cases flat backgrounds and 2 years of exposure are assumed.

\begin{tabular}{ccccc}
\hline \hline $\begin{array}{c}\text { Mass } \\
(\mathrm{kg})\end{array}$ & $\begin{array}{c}\text { Resolution } \\
(\mathrm{keV})\end{array}$ & $\begin{array}{c}\text { Threshold } \\
(\mathrm{keV})\end{array}$ & $\begin{array}{c}\text { Background } \\
(\mathrm{c} / \mathrm{kg} / \mathrm{keV} / \text { day })\end{array}$ & $\begin{array}{c}g_{a \gamma \gamma}^{\lim }(2 \text { years }) \\
\left(\mathrm{GeV}^{-1}\right)\end{array}$ \\
\hline 40.7 & 1 & 5 & 0.1 & $1.3 \times 10^{-9}$ \\
40.7 & 1 & 5 & 1 & $1.7 \times 10^{-9}$ \\
750 & 1 & 5 & 0.01 & $6.5 \times 10^{-10}$ \\
750 & 1 & 5 & 0.05 & $8.0 \times 10^{-10}$ \\
\hline \hline
\end{tabular}

Table 3: Expected limits on the photon-axion coupling for 2 years of exposure of CUORICINO and CUORE assuming the quoted values for the experimental parameters. These values will be valid throughout the axion model space for higher mass axions.

Finally, it should be noted that searches for other rare processes (like electron and nucleon stability, rare alpha and beta decays) are also possible in CUORE.

\section{Conclusions}

CUORE is a proposed array of one thousand, $750 \mathrm{~g}, \mathrm{TeO}_{2}$ bolometers operating at the Gran Sasso Underground Laboratory. It is intended to investigate rare events. The array has a cubic geometry of 25 towers of 40 detectors each. One such tower has been successfully constructed and is being operated in Gran Sasso as a test experiment, and also as an independent, first-step next generation DBD experiment called CUORICINO. With three months of data, a limit of $5.5 \times 10^{23}$ y has been derived at $90 \%$ CL for the half-life of neutrinoless $\mathrm{DBD}$ of ${ }^{130} \mathrm{Te}$. The corresponding upper bounds on the effective neutrino mass range from 0.37 to $1.9 \mathrm{eV}$ depending on the nuclear models.

Thus far, the CUORICINO performances imply that the expanded array, CUORE, will be a very competitive experiment. In fact, operating CUORICINO for 3 years with the present background level will yield a half-life sensitivity for neutrinoless double-beta decay of $6.1 \times 10^{24}$ years, corresponding to an effective mass of the electron neutrino of the order of $0.3 \mathrm{eV}$. CUORICINO data show us what and where the sources of background are and how to reduce them. We conclude that the required reduction in the background is possible. This would allow the full CUORE array to achieve a 5 -year sensitivity of $4.7 \times 10^{26}$ years, corresponding to an effective mass of the order of $30 \mathrm{meV}$. Another order of magnitude reduction will present a real challenge, but is possible. In that case, a 5-year sensitivity in the effective mass would be around $15 \mathrm{meV}$. In the least optimistic case above, and in the case of inverted hierarchy of the neutrino mass spectrum, a null experiment would strongly disfavour the Majorana character of neutrinos, whereas a definite observation would confirm that neutrinos are Majorana particles. Only double-beta experiments can achieve this goal. 


\section{Acknowledgments}

One of us (S. Cebrián) greatly acknowledges Spanish foundation "Fundación Areces" for the support of a fellowship at the Dipartamento di Fisica dell'Università di Milano-Bicocca.

\section{References}

[1] S. Fukuda et al., [SuperKamiokande Collaboration], 'P $\bar{P} y s$. Rev. Lett. $8 \overline{6}(200 \overline{1}) 565 \overline{6}$. M. B. Smy et al., arXiv:hep-ex/0309011. Q. R. Ahmad et al., [SNO Collaboration], Physs. Rev. Lett.' : - $89(2002) 0113011, P h y s$. Rev. Lett. $89 .(2002) 011302$. S. N. Ahmed et al.,

arXiv:nucl-ex/0309004. K. Eguchi et al., [KamLAND Collaboration], 'P्Phys. Rev. Lett.

: - -

[2] C. Arnaboldi et al., in press in Nucl. Instrum. Meth.A.

[3] E. Fiorini and T. Niinikoski, 'Nucl. Instrum. Meth. 224 (1984) 83 í.

[4] C. Arnaboldi et al., iPhys. Lett. B $557-2003) 167$;

[5] A. Alessandrello et al., iNucl. Instrum. Meth. A A12 (1998) $45 \overline{4}$.

[6] S. Pirro et al., Nivucl. Instrum. Meth. A $444(2000) 331$.

[7] A. Staudt, Kuo and H. Klapdor, Phys. Rev. $\bar{C}^{-} 46-(19 \overline{9} \overline{2})-\overline{8} \overline{1}$ i

[8] G. Pantis, F. Simkovic, J. D. Vergados, and A. Faessler, Physs. Rev.

[9] P. Vogel et al., 'Phys. Rev. Lett. 57 (1986) 3148, 'Phys.Rev.C 37 (1988) 73 . M. Moe and P.

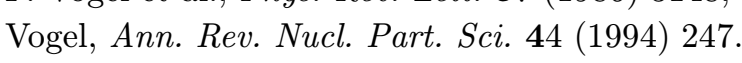

[10] O. Citivarese, A. Faessler, and T. Tomoda, 'Physs. Lett. B $1 \overline{9} \overline{4}(\overline{1} \overline{9} \overline{7}) \overline{1} 1$ A. Faessler, : Lett. B 199 (1987) 473 J. Suhonen and O. Citivarese, Phys. Rev C 49 (1994) 3055.

[11] T. Tomoda, 'Rep. Prog. Phys. $\mathbf{5} 4$ (1991) 53 i.

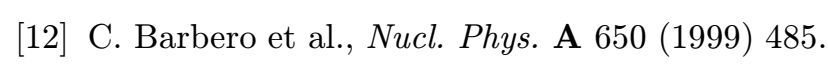

[13] F. Simkovich, iPhys. Rev.

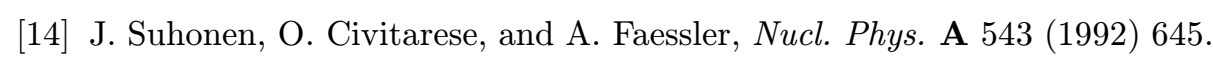

[15] K. Muto, E. Bender and H. V. Klapdor, 'Z. Phys. A

[16] S. Stoica and H. V. Klapdor, Phys. Rev. 63 (2001) 064304 .

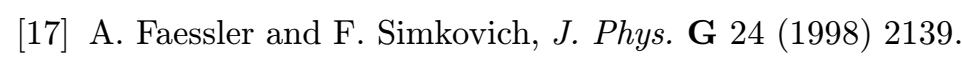

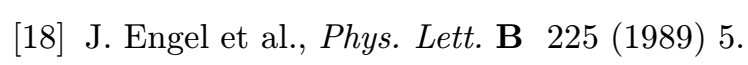

[19] M. Aunola and J. Suhonen, №vicl. Phys. A 643

[20] J. G. Hirsh et al, iNucl. Phys. A 589 (1995) $445_{\text {r. C. R. Ching et al, Phys. Rev. } \mathbf{C} 40(1984)}$ - $=304$. X. R. Wu et al, Phys. Lett. B 272 (1991) 169i. X. R. Wu etal, Phys. Lett. B 276 (1992)i $1=-274$

[21] C. Arnaboldi et al., 'Astropart. Physs.20 2003$) 91$

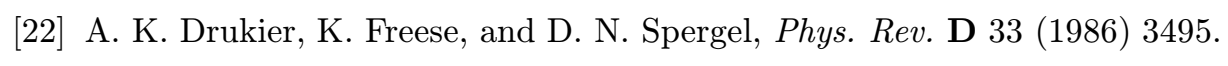

[23] R. Bernabei et al., [DAMA Collaboration], 'Riv. N. 
[24] D. Abrams et al., [CDMS Collaboration], Phys. Rev. D 66 (2002) 122003 . A. Benoit et al., [EDELWEISS Collaboration], Phys. Lett. B 545_(2002) 43. A. Morales et al., [IGEX

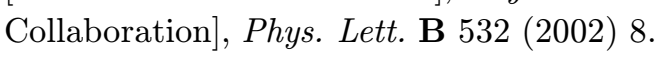

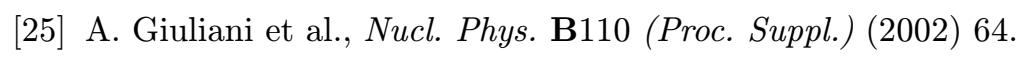

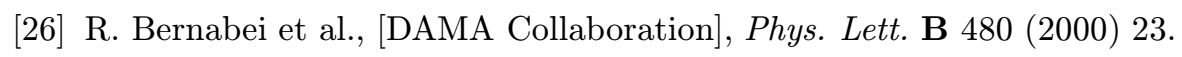

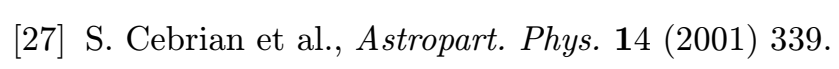

[28] R. J. Creswick et al., í

[29] F. T. Avignone et al., 'Phys. Rev. Lett. 81 (1998) 5068. A. Morales et al., 'Astropart. Phys. 16. - . - $(2002) 325$.

[30] R. Bernabei et al., 'Phys. Lett. B 515 _2001)66.

[31] S. Cebrian et al., 'Āstropart. Pिhys. $10 \overline{0}(\overline{1} 9 \overline{9}) \overline{3} 9 \overline{7}$.' 